\title{
Atividade física e envelhecimento: aspectos epidemiológicos
}

\author{
Sandra Mahecha Matsudo ${ }^{1,2}$, Victor Keihan Rodrigues Matsudo² e Turíbio Leite Barros Neto 3
}

\section{INTRODUÇÃO}

A relação entre atividade física, saúde, qualidade de vida e envelhecimento vem sendo cada vez mais discutida e analisada cientificamente. Atualmente é praticamente um consenso entre os profissionais da área da saúde que a atividade física é um fator determinante no sucesso do processo do envelhecimento. É o objetivo desta revisão estabelecer os principais fatores determinantes do nível de atividade física durante o envelhecimento e os benefícios do estilo de vida ativo na prevalência de doenças crônicas não transmissíveis, na mortalidade e na manutenção da capacidade funcional durante esse processo.

Alguns dos conceitos que serão utilizados ao longo da discussão dos assuntos aqui tratados têm sido adequadamente definidos e compilados pelos melhores especialistas da área, que em reunião especial chegaram a alguns consensos ${ }^{1}$. Dentre aqueles, os conceitos que merecem especial atenção são:

a) Atividade física: definida como qualquer movimento corporal produzido em conseqüência da contração muscular que resulte em gasto calórico.

b) Exercício: definido como uma subcategoria da atividade física que é planejada, estruturada e repetitiva; resultando na melhora ou manutenção de uma ou mais variáveis da aptidão física.

c) Aptidão física: é considerada não como um comportamento, mas uma característica que o indivíduo possui ou atinge, como a potência aeróbica, endurance muscular, força muscular, composição corporal e flexibilidade.

Desta forma poderíamos também considerar a própria definição dos autores ${ }^{1}$, de epidemiologia da atividade físi-

1. Coordenadora do Projeto Longitudinal de Envelhecimento e Aptidão Física de São Caetano do Sul.

2. Centro de Estudos do Laboratório de Aptidão Física de São Caetano do Sul - Celafiscs.

3. Cemafe - Escola Paulista de Medicina - Unifesp.

Recebido em: 15/10/2000.

Aceito em: 22/12/2000.

Endereço para correspondência:

Avenida Goiás, 1.400

09521-300 - São Caetano do Sul, SP

E-mail: celafiscs@celafiscs.com.br ca, como a parte da epidemiologia que se preocupa com: a) a associação entre os comportamentos da atividade física e a doença; b) a distribuição e determinantes dos comportamentos da atividade física em populações específicas; e c) a associação entre atividade física e outros comportamentos.

\section{Nível de atividade física, barreiras e motivação nos adul- tos de maior idade}

Dentre essas associações propostas pela epidemiologia da atividade física, têm surgido pesquisas tentando estabelecer o padrão do nível de atividade física em diferentes populações de indivíduos de maior idade. Em 1994, Caspersen et al. ${ }^{1}$ compilaram informação de cinco grandes levantamentos realizados na população do sexo masculino maior de 65 anos da Inglaterra, Estados Unidos e Holanda. De acordo com aquelas pesquisas, a caminhada foi uma das atividades mais realizadas, variando de $38 \%$ a $72 \%$, seguida pela jardinagem, que foi prevalente entre $37 \%$ e $67 \%$. Já atividades como correr, trotar, jogar tênis e golfe foram realizadas por menos que um em cada dez indivíduos.

Dados provenientes de 2.783 homens e 5.018 mulheres maiores de 65 anos de idade da Pesquisa Nacional de Saúde dos Estados Unidos de $1990^{2}$ determinaram a prevalência de atividade física regular, que naquele estudo foi definida como a participação em atividades físicas no tempo livre por três ou mais vezes por semana e por mais de 30 minutos nas últimas duas semanas. Com esses parâmetros os autores encontraram uma prevalência de atividade física regular de $37 \%$ no sexo masculino e $24 \%$ no sexo feminino. Mais uma vez, conforme os dados apresentados anteriormente, as atividades mais comumente realizadas foram a caminhada (69\% dos homens e $75 \%$ das mulheres) e a jardinagem (45\% dos homens e 35\% das mulheres).

O processo de avaliação diagnóstica do Programa Agita São Paulo realizado na Região Metropolitana e no Interior do Estado de São Paulo determinou o nível de conhecimento, barreiras e facilitadores à prática da atividade física em uma amostra de mais de 2.000 indivíduos maiores de 50 anos. De acordo com os dados encontrados por Andrade et al. ${ }^{3}$, constatou-se que as barreiras mais freqüentes para ambos os sexos em cidades pequenas do Interior do 
Estado foram: a) falta de equipamento; b) necessidade de repouso; c) falta de local; d) falta de clima adequado; e e) falta de habilidade. Quando analisamos as barreiras na Capital e nas cidades da Região Metropolitana encontramos que as barreiras mais frequientemente citadas foram: a) falta de equipamento; b) falta de tempo; c) falta de conhecimento; d) medo de lesão; e e) necessidade de repouso. Foi claramente evidenciado que as barreiras diferem segundo o sexo e o tamanho da cidade, como também são barreiras ligadas à condição de saúde e à vontade do indivíduo. No entanto, eram barreiras que poderiam ser superadas com a divulgação das novas mensagens de promoção da atividade física, que mostram como não há necessidade de equipamento, local, habilidade ou conhecimento para uma pessoa ser regularmente ativa.

Considerando os agentes facilitadores no ambiente para o envolvimento regular com a atividade física os dados de Andrade et al $^{3}{ }^{3}$ revelaram que, em ordem de prioridade, as pessoas maiores de 50 anos de idade realizavam atividade física por causa de indicação de:

\section{Sexo feminino:}

1) Orientação médica

2) Amigos

3) Familiares

4) Procura por companhia

5) Colegas de trabalho

6) Programa Agita São Paulo
$(38,3 \%)$

$(33,3 \%)$

$(10,4 \%)$

$(10,4 \%)$

$(4,2 \%)$

$(3,3 \%)$

\section{Sexo masculino:}
1) Orientação médica
2) Amigos
3) Procura por companhia
4) Programa Agita São Paulo
5) Familiares
6) Colegas de trabalho

$(50,3 \%)$

$(25,8 \%)$

$(7,0 \%)$

$(7,0 \%)$

$(6,3 \%)$

$(3,5 \%)$
Os dados claramente evidenciam a relevância do profissional da saúde, particularmente do médico, como vetor para o envolvimento regular com a atividade física nessa faixa etária. De forma similar, Yusuf et al. ${ }^{2}$ ressaltaram a importância da orientação médica sobre o nível de prática de atividade física de adultos e idosos como uma forma não só da prevenção primária das doenças crônicas, mas também da prevenção secundária em pacientes com doenças cardiovasculares e outras enfermidades.

Como parte de um estudo longitudinal de envelhecimento e aptidão física, Satariano et al. ${ }^{4}$ investigaram em 2.046 indivíduos maiores de 55 anos as barreiras para a prática da atividade física no tempo livre e encontraram que as mulheres reportaram mais barreiras para a prática do que os homens e as razões médicas incrementaram com a idade. As diminuições na velocidade de andar e sintomas de depressão figuram entre as razões não médicas citadas como obstáculos em homens e mulheres. Entre as primeiras cinco barreiras para a prática da atividade física nas mulheres foram citadas: a) a falta de companhia; b) a falta de interesse (mais comum nas mulheres de 65 a 74 anos); c) a fadiga; d) problemas de saúde; e e) a artrite. Nas mulheres maiores de 75 anos de idade problemas de saúde e funcionais, como medo às quedas, foram as principais barreiras mencionadas. Esses dados são similares aos já previamente reportados por Andrade et al. ${ }^{3}$ no levantamento feito no Estado de São Paulo. Desta forma a promoção da atividade física na terceira idade deve levar em consideração a falta de companhia e a falta de interesse, principais barreiras citadas, no momento de estabelecer políticas de saúde pública.

O nível de conhecimento em relação ao novo padrão da atividade física para a promoção da saúde mostrou ter um comportamento diferente de acordo com o sexo e o local analisado ${ }^{5}$. De acordo com o CDC/ACSM ${ }^{6}$ o novo paradigma da atividade física para a promoção da saúde recomenda que os indivíduos devem realizar atividade física de intensidade moderada, por pelo menos 30 minutos por dia, na maior parte dos dias da semana, de preferência todos, de forma contínua ou acumulada. Este diagnóstico foi realizado mediante a elaboração de um questionário aplicado em diversas cidades do Estado. Nas cidades pequenas o padrão de conhecimento sobre o novo paradigma da atividade física foi positivo em $32 \%$ da população feminina e em $35 \%$ da masculina. De forma similar, $36 \%$ das mulheres e $31 \%$ dos homens consideraram que a atividade física deveria ser feita de três a quatro vezes por semana e por mais de 30 minutos por sessão. Na Região Metropolitana e grandes cidades do Estado, o padrão foi relativamente melhor: praticamente $40 \%$ das mulheres e $51 \%$ dos homens responderam de acordo com o novo paradigma.

Mas os dados mais interessantes foram encontrados na relação entre o nível de conhecimento e o nível de atividade física da população idosa ${ }^{7}$. Nos dados das cidades mais populosas, menos de $23 \%$ daqueles que tinham conhecimento do novo paradigma praticavam atividade física de acordo com essas novas recomendações. Entre 25\% (sexo masculino) e $32 \%$ (sexo feminino) deles eram irregularmente ativos, enquanto $48 \%$ das mulheres e $53 \%$ dos homens eram sedentários. Nas cidades menores os dados são mais preocupantes: somente $14 \%$ dos indivíduos que têm conhecimento do novo paradigma praticam atividade física de acordo com estas recomendações. No sexo feminino, $34 \%$ eram irregularmente ativas e $41 \%$ sedentárias. No 
masculino, $25,2 \%$ eram irregularmente ativos e praticamente $40 \%$ sedentários.

Estes dados corroboram a premissa de que o nível adequado de conhecimento não necessariamente reflete em um envolvimento regular com atividade física de acordo com os novos paradigmas. Assim, a promoção da atividade física nesta população deveria enfatizar não somente o incremento no nível de conhecimento, mas também as estratégias para superar as barreiras, o que facilitaria a adoção de um estilo de vida ativo. Estas afirmações vão de acordo com aquelas postuladas por Yusuf et al. ${ }^{2}$, que encontraram na população analisada um nível adequado de conhecimento em relação ao exercício, mas ao mesmo tempo essas pessoas consideravam importantes as barreiras relacionadas ao conhecimento. Tal fato poderia explicar, como encontrado nos nossos levantamentos, por que muitos idosos acreditam na necessidade de diminuir o exercício com o passar da idade ou crer que conseguem um nível adequado de atividade física com as suas rotinas diárias de atividades.

Os dados encontrados nas avaliações feitas no Estado de São Paulo, anteriormente mencionados, diferem em termos de gênero do nível de atividade física encontrado por Yusuf et al. ${ }^{2}$, que relataram menor prevalência de mulheres regularmente ativas do que homens. Oyama e Oliveira ${ }^{8}$ consideram também que não existiu diferença na adaptação ao meio entre idosos (homens e mulheres) que participaram de programas de educação física. Com o objetivo de analisar os padrões de atividade física durante o curso da vida em mulheres idosas ativas e sedentárias, Cousins e Keating ${ }^{9}$ encontraram que eventos similares aconteceram na vida desses dois grupos de mulheres. No entanto, as mulheres ativas deram um destino diferente aos desafios e começaram ou mantiveram a prática da atividade física, enquanto que as inativas responderam de forma diferente tentando "conservar energia" e assim reduziram ou pararam o movimento. Em uma abordagem similar, Young et $a l .{ }^{10}$ analisaram os fatores determinantes do nível de exercício de 2.668 indivíduos de 50-65 anos de idade, que foram classificados como sedentários (7,8\% homens e 12,2\% mulheres), pouco ativos $(76,7 \%$ homens e $72,3 \%$ mulheres) e regularmente ativos ( $15,5 \%$ homens e mulheres). Os resultados sugerem que os homens solteiros, mulheres que reportaram pouca saúde, fumantes e indivíduos com sobrepeso foram os grupos com maior probabilidade de ser completamente sedentários. Da mesma forma, os sedentários tiveram maior prevalência de hipertensão arterial, acidente vascular cerebral e limitações físicas. As taxas de aderência a um programa de um ano de exercício foram menores entre aqueles que eram inicialmente sedentários $(39,5 \%)$ do que naqueles pouco ativos $(71,6 \%)$, mas au- mentaram nos programas de baixa intensidade realizados em casa em comparação com aqueles que usaram exercícios de alta intensidade (em grupo ou em casa), o que é de grande relevância na hora de formular estratégias de promoção de atividade física em grupos de idosos. Neste caso a promoção do novo paradigma, já aqui postulado ${ }^{6}$, de atividades físicas moderadas, convenientes e ligadas à rotina diária pode ser o mais efetivo para os grupos de idosos sedentários.

\section{Programas de atividade física na terceira idade}

Um dos aspectos que devem ser considerados na relação atividade física, doença e saúde em termos populacionais é a escolha do tipo de atividade física a ser prescrito na terceira idade. Recentemente têm surgido recomendações específicas de programas de atividade física para atender a população de adultos maiores de 60 anos de idade, tais como as normas propostas pela Organização Mundial da Saúde ${ }^{11}$ ou o Programa de Promoção da Atividade Física: Agita São Paulo na população do Estado de São Paulo (Governo do Estado de São Paulo ${ }^{12}$ ). De acordo com novos paradigmas e evidências, Ettinger et al. ${ }^{13}$, sumarizando os critérios de realização de testes em indivíduos idosos, sugeriram a necessidade dos mesmos somente para indivíduos acima de 50 anos nos casos de exercícios vigorosos. No caso de exercícios moderados, somente deveriam ser submetidos a testes de esforço aqueles com doenças crônicas, ou com presença de fatores de alto risco e ainda aqueles sintomáticos.

Embora o Colégio Americano de Medicina Esportiva recomende um teste de esforço para todo indivíduo acima de 50 anos que queira começar um programa de treinamento vigoroso, esta recomendação provavelmente não será necessária para indivíduos idosos que simplesmente queiram caminhar ou participar de um programa de treinamento de resistência, como sugerido recentemente por Evans ${ }^{14}$. Esse autor recomenda nestes casos a aplicação de um questionário simples para identificar os sujeitos que precisam de uma avaliação médica. Esta conclusão foi consequiência de sua experiência com o programa Keep Moving - Fitness after 50, que envolvia mais de 8.000 homens e mulheres em programas de caminhada, no qual não foram registrados casos de morte durante a atividade física.

\section{Atividades aeróbicas, treinamento de força muscular $e$ tai chi chuan}

Quanto ao tipo de atividade aeróbica a ser realizada, é recomendada a prescrição de atividades de baixo impacto, como a caminhada, o ciclismo ou pedalar na bicicleta, a natação, a hidroginástica, o remo, subir escadas, dançar, ioga, tai chi chuan e dança aeróbica de baixo impacto. Es- 
tas atividades são preferíveis àquelas chamadas de alto impacto, como o jogging, a corrida, esportes que envolvam saltos, como o vôlei e o basquete, pular corda e a dança aeróbica de alto impacto, que acarretam grande incidência de lesões nessa época da vida. A caminhada é com certeza a atividade física ideal para os indivíduos idosos que resulta em importantes efeitos benéficos para a saúde por ser uma atividade que sustenta o peso corporal e de baixo impacto, que pode ser feita em diferentes intensidades, em qualquer local, envolvendo grandes grupos musculares, contribuindo para aumentar o contato social, principalmente em grupos em risco de isolamento, depressão e demência $^{15}$. Neste aspecto, de acordo com recente metanálise realizada por Arent et al. ${ }^{16}$, o exercício está associado com melhora significante do humor em pessoas idosas, sendo que os efeitos têm sido encontrados com qualquer tipo de exercício, mas em especial com o treinamento de força muscular, realizado em intensidade leve a moderada. Da mesma forma, a melhora do condicionamento aeróbico parece estar associada em indivíduos que envelhecem com melhor função neurocognitiva ${ }^{17}$.

No Brasil existem alguns programas de educação física especificamente direcionados à terceira idade. Desde 1994 o Programa para Autonomia da Atividade Física (PAAF), da Faculdade de Educação Física da USP, visa dar condições ao idoso de autogerir um programa de atividade física. O programa se constitui de atividades teóricas e práticas em duas sessões semanais de 90 minutos, durante 18 meses, em que são desenvolvidas: a reestruturação corporal, atividades de relaxamento e respiratórias, atividades aeróbicas e atividades direcionadas à força muscular, flexibilidade, controle motor, locomoção, equilíbrio e ritmo ${ }^{18}$. Da mesma forma, o esquema IMMA (Idosos em Movimento Mantendo a Autonomia), da Universidade do Estado de Rio de Janeiro, é um programa que inclui aulas de ginástica e dança objetivando trabalhar a flexibilidade, mobilidade articular, equilíbrio estático e dinâmico, coordenação, deslocamento e relaxamento. Desta forma as aulas são elaboradas obedecendo a um esquema pedagógico mais flexível às alterações do envelhecimento ${ }^{19}$.

Um aspecto fundamental do programa de exercício é o fortalecimento da musculatura procurando incrementar a massa muscular e, por conseguinte, a força muscular, evitando assim uma das principais causas de inabilidade e de quedas. Além disso, a massa muscular é o principal estímulo para incrementar a densidade óssea. Recentemente têm sido estabelecidas ${ }^{14,20}$ as normas de prescrição do treinamento de resistência na população idosa, incluindo até os grupos de hipertensos e pacientes com artrite reumatóide e osteoartrite. De acordo com essas recomendações o treinamento de resistência deve estar dirigido aos grandes grupos musculares que são importantes nas atividades da vida diária. Entretanto, qualquer tipo de sobrecarga pode e deve ser utilizado nos programas de força muscular na população acima de 50 anos. Assim pode-se fazer uso de qualquer peso confeccionado com tecido, areia e velcro para ser colocado nos membros superiores e inferiores, ou ainda o simples uso de garrafas, latas, sacos ou qualquer objeto doméstico com água, areia, feijão, que viabilizariam o treinamento de resistência ${ }^{21}$. Os benefícios encontrados nestes pacientes incluem: aumento da força dinâmica, do pico da capacidade de exercício, da endurance submáxima, diminuição dos valores de percepção subjetiva de esforço durante exercício intenso e relatos de melhora da função nas atividades vigorosas da vida diária. As evidências, embora limitadas, deste tipo de treinamento na auto-eficácia de pacientes com doença coronariana sugerem que este tem um impacto positivo nas tarefas relacionadas à força $\mathrm{e}$ na melhora da qualidade de vida ${ }^{22,23}$. Segundo Fiatarone ${ }^{24}$, o treinamento de força muscular deve ser preferido ao treinamento aeróbico nas seguintes circunstâncias: artrite grave, inabilidade para suportar o peso corporal, ulcerações no pé, desordens do equilíbrio, amputação, doença pulmonar obstrutiva crônica e baixo limiar para isquemia. Embora sejam recomendados para a população idosa tanto os programas de atividades aeróbicas como os de treinamento de força muscular, são estes últimos que realmente podem parar ou reverter de alguma forma a perda de massa muscular, sendo portanto as atividades de preferência na manutenção da capacidade funcional e independência ${ }^{14}$.

$\mathrm{Na}$ escolha das atividades aeróbicas ou de treinamento de força muscular vários aspectos devem ser considerados, mas especialmente os objetivos a serem atingidos. As evidências disponíveis e amplamente analisadas por Hurley e Hagberg ${ }^{25}$ indicam claramente que tanto o treinamento aeróbico quanto o treinamento de força no idoso têm efeitos benéficos. Os autores sintetizam que os dois tipos de treinamento melhoram a densidade mineral óssea, a homeostase da glicose e o risco de queda; porém, se o objetivo for melhorar o condicionamento cardiovascular, diminuir a hipertensão arterial, melhorar o perfil de lipoproteínas plasmáticas ou amenizar a hipertrofia do ventrículo esquerdo, o treinamento aeróbico parece ser o método mais eficaz. Por outro lado, se o objetivo for aumentar a massa e a força muscular, o treinamento de resistência é a melhor opção. No entanto, nenhum dos tipos de treinamento tem mostrado piorar algum dos parâmetros-chaves cardiovasculares e músculo-esqueléticos. Dessa forma, concordamos integramente com a posição dos autores que a melhor opção para o indivíduo que está envelhecendo é a realização de um programa de atividade física que inclua tanto o treinamento aeróbico como o de força muscular e que ainda 
incorpore exercícios específicos de flexibilidade e equilíbrio.

Apesar de ser uma arte marcial chinesa milenar, que encoraja a concentração mental e o controle dos movimentos corporais, foi somente nos últimos anos que começaram a surgir as primeiras publicações científicas sobre os efeitos do tai chi chuan na aptidão física e capacidade funcional do idoso. Essa atividade representa uma alternativa de atividade efetiva e funcional, particularmente para os idosos que freqüentemente estão em risco por causa de alguns problemas associados ao envelhecimento, como artrite, disfunção neurológica, declínio geral do equilíbrio, coordenação e função locomotora. Isso se deve a sua natureza de respeito ao ritmo da própria pessoa, não estressora e não competitiva, assim como sua habilidade de economizar tempo, espaço e equipamento ${ }^{26}$. As evidências científicas têm mostrado efeitos benéficos significativos da prática regular do tai chi chuan no equilíbrio e nos movimentos suaves dos braços ${ }^{27}$, no consumo máximo de oxigênio, na flexibilidade toracolombar, na força muscular de extensão e da flexão do joelho ${ }^{28}$, assim como na função cardiorrespiratória de pacientes com cirurgia de revascularização cardía$\mathrm{ca}^{29}$. No nosso grupo de pesquisa, Oliveira et al. ${ }^{30}$ analisaram os efeitos de três meses de um programa de tai chi chuan praticado uma vez só por semana e mesmo assim verificaram efeitos significantes na diminuição de gordura corporal e no aumento da força muscular dos membros inferiores, equilíbrio e flexibilidade do tronco. Os mesmos autore $^{31}$ analisaram o efeito de um período de 12 semanas de destreinamento na aptidão física do mesmo grupo, não encontrando diferenças significativas nas variáveis analisadas no período.

No entanto, os efeitos benéficos do tai chi chuan têm sido demonstrados também em variáveis relacionadas à saúde, como é o caso do estudo realizado por Young et $a l .{ }^{32}$ com idosos sedentários hipertensos, encontrando uma redução estatisticamente significante na pressão arterial sistólica e diastólica. O conjunto de informações científicas recentes permite concluir que o tai chi chuan pode ser uma grande alternativa em termos de saúde pública para controlar ou reduzir a pressão arterial, assim como para melhorar outras variáveis da aptidão física relacionadas à saúde e à qualidade de vida de indivíduos idosos.

\section{Promoção do estilo de vida ativo}

Os programas de promoção da atividade física na comunidade para indivíduos acima de 50 anos de idade têm crescido em popularidade nos últimos anos. Considerando as novas propostas internacionais de atividade física como forma de promover saúde na população, surgiu o Programa Agita São Paulo, que tem como objetivo aumentar o nível de conhecimento da população sobre os benefícios da atividade física e aumentar o nível de atividade física da população do Estado de São Paulo ${ }^{33}$. Um dos focos principais do programa é a população da terceira idade e a proposta de prescrição de atividade para essa população é realizar atividades físicas de intensidade moderada, por pelo menos 30 minutos por dia, na maior parte dos dias da semana, se possível todos, de forma contínua ou acumulada. O mais importante deste novo conceito é que qualquer atividade da vida cotidiana é válida e que as atividades podem ser realizadas de forma contínua ou intervaladas, ou seja, o importante é acumular durante o dia 30 minutos de atividade ${ }^{34}$.

Uma das mais relevantes contribuições feitas nos últimos anos pelo Colégio Americano de Medicina Esportiva na área da atividade física e o envelhecimento tem sido o recente posicionamento oficial sobre exercício e atividade física para o idoso ${ }^{23}$. Os mais renomados especialistas da área concluem que: a) participação em um programa de exercício regular é uma modalidade de intervenção efetiva para reduzir e/ou prevenir alguns dos declínios associados com o envelhecimento; b) o treinamento aeróbico é efetivo para manter e melhorar as funções cardiovasculares e, portanto, o desempenho físico, assim como tem um papel fundamental na prevenção e tratamento de diversas doenças crônico-degenerativas, contribuindo para aumentar a expectativa de vida. O treinamento de força, por sua parte, está relacionado, de acordo com os autores, com a compensação na perda da massa e força muscular, melhorando a capacidade funcional e conseqüentemente a qualidade de vida.

Os autores recomendam naquele documento ênfase ao treinamento do equilíbrio (embora as evidências científicas neste aspecto não sejam ainda suficientes), trabalhando com posturas progressivamente mais difíceis que reduzem a base de apoio, que necessitem de movimentos dinâmicos que ativem o centro de gravidade e estresse de grupos musculares posturalmente importantes. No idoso frágil ou debilitado, no entanto, a prescrição mais difícil é a do treinamento aeróbico devido à presença de algumas condições clínicas, como desordens do andar, artrite, demência, doenças cardiovasculares, problemas ortopédicos e dos pés, incontinência e alterações visuais. Nessas circunstâncias a prioridade é o treinamento de força e do equilíbrio que garantam as condições para iniciar um programa de caminhada. Nos casos em que esta atividade não seja possível, podem ser recomendadas atividades como ergômetro de braços, de pernas ou exercícios sentados dentro da água. Neste sentido, um ponto em comum entre esse posicionamento oficial ou de outros pesquisadores da área ${ }^{24,36,37}$ como também da nossa própria visão da ativida- 
de física para os indivíduos idosos são as variáveis que devem ser priorizadas para prescrever atividade física na terceira idade para manter a independência funcional do indivíduo: a) força muscular; b) equilíbrio; c) potência aeróbica; d) movimentos corporais totais; e e) por último, mas não menos importante, as mudanças do estilo de vida, sem as quais não acreditamos que seja possível manter na totalidade a capacidade funcional do idoso.

\section{Atividade física, mortalidade e longevidade}

Um dos aspectos mais fascinantes que tem sido motivo de várias pesquisas é a relação entre o exercício, atividade física e a longevidade. Alguns dados obtidos com atletas mostraram que, apesar de não continuarem realizando atividade física de forma sistemática, eles possuem maior capacidade física ( $\mathrm{V}_{2}$ máx.) do que seus companheiros não-atletas sedentários, possivelmente pelos altos padrões de atividade de lazer destes ex-atletas ${ }^{38}$. A partir dos tradicionais estudos realizados por Paffenbarger ${ }^{39} \mathrm{com}$ o seguimento de aproximadamente 16.000 ex-alunos de Harvard por 16 anos, foi observado que os indivíduos que pararam de praticar esportes tiveram $35 \%$ de incremento no risco de morte sobre aqueles que continuaram sedentários, provavelmente porque as condições pré-mórbidas causaram diminuição da atividade e morte precoce. Porém, aqueles que começaram a praticar esportes experimentaram um índice $21 \%$ menor de morte que aqueles habitualmente sedentários. Aqueles que se tornaram mais vigorosos experimentaram um índice $28 \%$ menor de morte que aqueles que sempre se mantiveram vigorosos, um índice $37 \%$ menor que os que nunca fizeram exercícios vigorosos. Com a mesma amostra dividida em três grupos de acordo com a energia gasta em atividades como caminhar, subir escadas e praticar esportes, o autor achou um incremento na expectativa de vida maior nos indivíduos que eram mais jovens quando entraram no estudo e como também nos mais ativos $(2.000 \mathrm{cal} / \mathrm{sem})$, quando comparados com os menos ativos $(500 \mathrm{kcal} / \mathrm{sem})$ e moderadamente ativos $(501$ $1.999 \mathrm{kcal} / \mathrm{sem})$. Quando os mais ativos foram comparados com os menos ativos, o aumento na expectativa de vida foi em média de 2,51 anos para indivíduos de 35-39 anos de idade no início do estudo e de 0,42 anos nos indivíduos de 75-79 anos. Um dado também interessante foi o fato de que a percentagem de indivíduos maiores de 80 anos foi maior nos indivíduos mais ativos $(69,7 \%)$ do que nos menos ativos $(59,8 \%)$.

Em estudo similar publicado na mesma época, Blair et $a l .{ }^{40}$, analisando uma amostra de mais de 13.000 homens e mulheres seguidos durante oito anos, encontraram, como Paffenbarger ${ }^{39}$, uma associação inversa forte, gradativa e consistente entre aptidão física e mortalidade em homens e mulheres. Foram observadas menores taxas de mortalidade total, assim como por doenças cardiovasculares e câncer em indivíduos com melhores níveis de condicionamento físico. Alguns anos depois, utilizando a mesma amostra dos alunos de Harvard do estudo de Paffenbarger, Lee et $a l .^{41}$ analisaram em indivíduos do sexo masculino as associações entre a atividade física vigorosa e não vigorosa com a longevidade. Evidenciaram nesse caso que a relação inversa entre a atividade física e longevidade somente foi encontrada com as atividades físicas vigorosas (> 6 MET: taxa metabólica de repouso).

Por outro lado, dados provenientes de uma amostra de mulheres suecas avaliadas em um intervalo de $20 \operatorname{anos}^{42}$ evidenciaram que a diminuição nos níveis de atividade física, assim como baixos níveis iniciais de atividade física, foram um forte fator de risco de mortalidade. Assim, os autores sugerem que a manutenção de um adequado nível de atividade física é um importante fator de promoção da saúde na população idosa. Um dos maiores estudos feitos com mulheres foi o realizado com 40.417 pós-menopáusi$\operatorname{cas}^{43}$ seguidas por sete anos. Foi encontrado que aquelas que reportaram atividade física regular tiveram um risco significativamente menor de morte (30\%) quando comparadas com as mulheres que não reportaram atividade física. O menor risco de morte foi evidente tanto para o risco por doenças cardiovasculares como por enfermidades respiratórias, sendo menor quanto maior o nível de atividade física. Um fato interessante encontrado naquele estudo e que não corrobora outros autores aqui expostos foi que o benefício também se observou entre aquelas mulheres que realizavam atividades físicas moderadas uma vez por semana.

Dois dos autores mais reconhecidos nesta área, Paffenbarger e Lee, realizaram em $1996^{44}$ uma das mais extensas revisõ es sobre a relação entre atividade física, aptidão física e longevidade, analisando criticamente cada uns dos trabalhos feitos até então nesta área do conhecimento. De acordo com os dados apresentados pelos autores, os sujeitos com altos níveis de atividade física e aptidão física experimentaram menor risco de doenças cardiovasculares e viveram mais (em torno de dois anos) assim como aqueles que adotaram um estilo de vida ativo. No entanto, a maioria destes dados provém de estudos observacionais e não randomizados. Outros estudos analisados pelos autores mostraram que a atividade física também tem um impacto positivo em variáveis intermediárias, como a pressão arterial, o perfil de lipoproteínas e a tolerância à glicose, que influenciam a saúde e a longevidade. De forma similar, Farrell et al. ${ }^{45}$, com 25.341 pacientes do sexo masculino seguidos em média por 8,4 anos e classificados em três níveis de condicionamento físico (alto, médio e baixo), observaram uma forte associação inversa entre o nível de 
condicionamento e mortalidade por doenças cardiovasculares em indivíduos sem outros fatores de risco (fumo, colesterol e pressão arterial elevadas), mas também em indivíduos fumantes e com colesterol elevado. Não foram encontradas associações em hipertensos ou sujeitos com dois ou mais fatores de risco.

Dando continuidade à análise da mais importante fonte de dados sobre fatores de risco, mortalidade e longevidade (os alunos de Harvard analisados consecutivamente nos anos 60, 70, 80 e 90), foram avaliados ${ }^{46}$ os dados das taxas de mortalidade; encontrou-se que os homens que caminharam menos de $15 \mathrm{~km}$ por semana, subiram menos de 20 andares por semana ou não participaram em esportes moderadamente vigorosos tiveram $10 \%, 20 \%$ e $38 \%$ maior risco de morte, respectivamente, do que aqueles indivíduos que realizaram estas atividades em uma intensidade maior de 4,5 MET. Para explorar a hipótese de que as mudanças favoráveis na atividade física poderiam melhorar a qualidade de vida e retardar a mortalidade, os autores analisaram essas mudanças nos alunos e encontraram reduções evidentes na mortalidade com o incremento no nível de atividade física e aumentos gradativos na mortalidade com a diminuição no nível de atividade física. Segundo os cálculos feitos, o ganho em expectativa de vida pela mudança do estilo de vida ativo foi de 1,5 ano. Conclusões similares foram feitas por Kushi et al..$^{43}$ com mulheres, em que o incremento no nível de atividade física, ou seja, aquelas que nunca ou raramente se engajavam em atividade física regular e que passaram a fazer atividade pelo menos quatro vezes por semana, foi associado significativamente com uma redução no risco de morte.

Evidências epidemiológicas mais recentes ${ }^{47}$ em 5.567 homens de 40 a 59 anos de idade sustentam mais uma vez que o hábito de realizar atividades físicas leves ou moderadas reduz a taxa de mortalidade total e a de mortalidade por causa cardiovascular em homens de idade avançada. Já com dados de uma amostra bem menor, Sihvonen et $a l .^{48}$ evidenciaram decréscimo no nível de atividade física entre os 75 e 80 anos de idade e uma associação significante entre atividade física e melhor sobrevivência no grupo de mulheres de 80 anos e dos homens de 75 anos. De forma similar, os achados de um grupo de homens seguidos por 12 anos $^{49}$ demonstraram que as taxas de mortalidade foram quase duas vezes superiores nos homens que caminhavam menos de $1,6 \mathrm{~km}$ por dia, do que naqueles que andavam mais do que $3,2 \mathrm{~km}$ diários. A distância caminhada nesse caso permaneceu inversamente relacionada à mortalidade, mesmo quando ajustada a outras medidas de atividade e outros fatores de risco. De acordo com os dados de um dos últimos estudos realizados com os ex-alunos de Harvard, Lee e Paffenbarger ${ }^{50}$ verificaram em uma amostra de 13.485 homens que a distância caminhada e as escadas subidas predisseram a longevidade independentemente, mas as atividades leves não foram associadas com menores taxas de mortalidade. No entanto, os autores concluem que parece ser que as atividades físicas de alguma forma são benéficas e as vigorosas claramente predizem taxas baixas de mortalidade. Em estudo mais recente, Lee et al. ${ }^{51}$ utilizaram uma coorte prospectiva de 13.905 homens do tradicional estudo de alunos de Harvard para verificar a associação entre atividade física e risco de câncer. Ajustando os resultados para idade, fumo e índice de massa corporal, atividades como caminhar, subir escadas e realizar atividades físicas de intensidade moderada (mais de 4,5 MET) foram inversamente relacionadas com o risco de câncer de pulmão. No entanto, esta associação não foi encontrada com as atividades leves. Desta forma, os dados indicam que provavelmente seis a oito horas por semana de atividade física de intensidade moderada podem significativamente diminuir o risco de câncer de pulmão em homens. Esses dados trazem uma nova perspectiva, pois até então essa associação não tinha sido verificada.

Estudos longitudinais mais recentes têm verificado a associação entre atividade física e risco de doenças cardiovasculares, principal causa de mortalidade na população $0^{52,53}$. A caminhada mostrou novamente ser um fator protetor importante de risco de doenças cardiovasculares ${ }^{52}$. Embora não tenha sido encontrada uma associação entre a atividade física total (gasto energético total caminhando, subindo escadas e praticando esportes) e o risco de doenças cardiovasculares em mulheres, caminhar dez ou mais quarteirões por dia (em torno de $9,7 \mathrm{~km}$ por semana) foi associado com uma diminuição de $33 \%$ no risco. De forma similar, Mensink et al. ${ }^{53}$, estudando aproximadamente 11.000 homens e mulheres de 50 a 69 anos de idade, encontraram que os níveis atualmente recomendados de atividade física (cinco ou mais vezes e 3,5 horas ou mais por semana) foram associados com menor prevalência de fatores de risco para doenças cardiovasculares como hipertensão arterial e aumento do índice de massa corporal.

Relevantes dados epidemiológicos em relação à atividade física e o risco de acidente vascular cerebral (AVC) foram recentemente publicados por Evenson et al. ${ }^{54}$, mostrando que a prevalência deste problema foi maior nos indivíduos classificados em menor nível de atividade física realizada, como esporte, no trabalho e no tempo livre. Mas essa relação foi considerada como fraca pelos autores. Por outro lado, analisando prospetivamente a relação entre atividade física e acidente vascular cerebral (AVC), Hu et al..$^{55}$, seguindo durante oito anos mais de 72.000 enfermeiras de 40 a 65 anos de idade, mostraram valores mais consistentes de associação entre essas duas variáveis. $\mathrm{O}$ incremento 
no nível de atividade física foi associado significantemente com a diminuição no risco de $\mathrm{AVC}$ isquêmico (não para o AVC hemorrágico), mesmo quando controlado em relação à idade, índice de massa corporal e história de hipertensão. Porém, os dados mais interessantes foram que a caminhada rápida mostrou o mesmo nível de associação com a diminuição do risco de AVC do que a atividade física vigorosa e que as mulheres sedentárias que se tornaram ativas na meia-idade ou no final desta apresentaram menor risco de AVC do que aquelas que permaneceram sedentárias. Esse estudo é, portanto, uma das grandes evidências de que a inclusão de uma atividade tão popular e econômica como a caminhada pode ter um impacto significante na redução do risco de acidente vascular cerebral em mulheres, sendo assim uma estratégia prática em termos de saúde pública para controlar uma das principais causas de morte.

Com base em todos os dados expostos anteriormente podemos inferir que as evidências epidemiológicas disponíveis, tanto em homens quanto em mulheres, sugerem fortemente uma associação inversa entre atividade física e mortalidade (principalmente por doenças cardiovasculares), sendo que esta associação é mais forte com o nível de atividade física atual. Desta forma, os dados suportam a necessidade do estímulo à atividade física regular após os 50 anos, mesmo que o indivíduo seja sedentário, visto que é a manutenção da atividade física regular ou a mudança a um estilo de vida ativo que tem um impacto real na mortalidade e longevidade.

\section{Atividade física, capacidade funcional e mobilidade}

Um dos conceitos mais relevantes ao tratar do envelhecimento e sua relação com a saúde, a aptidão física e a qualidade de vida é sem dúvida o da capacidade funcional, definido também como estado funcional. Dados apresentados por Brill et al..$^{56}$ avaliando 3.069 homens e $589 \mathrm{mu}-$ lheres de 30 a 82 anos de idade durante cinco anos revelaram que nesse período $7 \%$ dos homens e $12 \%$ das mulheres reportaram pelo menos uma limitação funcional, que foi mensurada pela capacidade de realizar atividades da vida diária, atividades domésticas e de cuidado pessoal, assim como atividades leves, moderadas e vigorosas no tempo livre. No entanto, quando a limitação funcional foi analisada levando em consideração o nível de força muscular, os sujeitos que apresentaram maiores níveis mostraram também menos prevalência de limitações funcionais. Esse estudo se converte em mais uma evidência científica da importância da força muscular, na meia-idade e na terceira idade, na manutenção e/ou menor declínio da capacidade funcional com o envelhecimento.

Lee $^{57}$, estudando em um período de seis anos um pouco mais de 7.500 sujeitos de mais de 70 anos de idade, mediu o impacto da auto-avaliação da saúde geral como fator determinante do declínio funcional e da mortalidade. De forma geral, os riscos de declínio na capacidade funcional e de mortalidade foram maiores nos idosos que avaliaram sua saúde geral, física e mental de forma menos favorável. Uma das mais interessantes conclusões daquele estudo foi que a habilidade do indivíduo tomar cuidado de si é um fator preditivo significante e independente de mortalidade e capacidade funcional.

Com um objetivo similar ao anteriormente apresentado, Laukkanen et al. ${ }^{58}$ descreveram, como parte do estudo longitudinal realizado com mais de 600 homens e mulheres de 75 anos e 80 anos de idade seguidos durante cinco anos, o papel da saúde e da capacidade funcional como fatores preditivos da habilidade do idoso de viver em sua própria casa. As variáveis medidas incluíram a capacidade funcional, pela auto-avaliação da realização de 10 atividades da vida diária (AVD), força muscular, velocidade de andar, capacidade cognitiva e estado de saúde. A avaliação longitudinal do papel preditivo das dificuldades na realização das AVD evidenciou que a necessidade de ajuda para realizar uma ou mais das 10 AVD predizia significativamente a necessidade de cuidado institucional. As diferenças foram mais acentuadas no primeiro ano, mas mesmo após cinco anos aqueles que reportaram não necessitar de ajuda na avaliação inicial apresentaram maior tendência a não precisar de ser institucionalizados e ao mesmo tempo menores taxas de mortalidade. Com relação ao estado de saúde, o valor preditivo foi mais pronunciado. Nos indivíduos de 75 anos que não tiveram nenhuma doença diagnosticada no início, foi observado que após um ano não houve necessidade de receber apoio institucional, enquanto que naqueles com diagnóstico de uma doença, $40 \%$ entraram em uma instituição. Esses dados longitudinais reforçam a importância da manutenção da capacidade funcional e da prevenção das doenças não somente como mecanismo de diminuir as taxas de mortalidade, mas como forma de minimizar o risco de institucionalização nos últimos anos de vida.

Outras evidências neste aspecto foram previamente apresentadas por Leveille et al..$^{59}$ que, seguindo por 10 anos um pouco mais de 1.000 homens e mulheres acima de 65 anos, verificaram que um alto nível de atividade física aos 65 anos de idade foi associado com um maior nível de sobrevivência aos 80 anos de idade. Em torno de $63-70 \%$ daqueles que mantiveram um alto nível de atividade física sobreviveram aos 85 anos, enquanto somente $34-47 \%$ daqueles com baixo nível conseguiram sobreviver naquela idade. Os indivíduos que se mantiveram mais fisicamente ativos tiveram duas vezes mais chances de morrer sem incapacidade comparados com os sedentários. 
Com o propósito de determinar a relação entre os fatores de risco para a saúde e a incapacidade, Vita et al. ${ }^{60}$ estudaram em torno de 1.700 alunos longitudinalmente durante 24 anos, analisando a mortalidade e fatores como tabagismo, obesidade (pelo índice de massa corporal) e o nível de atividade física (minutos de atividade física vigorosa por semana). Os indivíduos que tiveram mais fatores de risco apresentaram o dobro do índice de incapacidade do que aqueles com baixo índice de risco. Um fato interessante observado foi que o início da incapacidade foi retardado em mais de cinco anos no grupo de baixo risco comparado com o de alto risco. Desta forma, foi verificado que o exercício, o fumo e o índice de massa corporal na meiaidade e no final da idade adulta são preditores da incapacidade; e o mais importante é que não somente as pessoas que têm melhores hábitos de saúde vivem mais, mas, além disso, nesses indivíduos a incapacidade é retardada e limitada a alguns anos no final da vida.

Analisando este tópico, Morey et al. ${ }^{61}$ verificaram a relação entre idade, sexo, raça, nível educacional, índice de massa corporal, depressão, número de doenças e consumo máximo de oxigênio com a função física em adultos de 65 a 90 anos de idade. Constataram que a variável com a correlação mais forte com a capacidade funcional foi o consumo máximo de oxigênio, sendo que o valor de $18,3 \mathrm{ml} . \mathrm{kg}^{-1}$. $\min ^{-1}$ foi considerado como o limite entre o baixo e o alto nível de função física. Por essa razão, aqueles com valores menores que este limite apresentaram maior dificuldade na realização de tarefas da vida diária. Nesse sentido, em outro estudo longitudinal ${ }^{62}$ conduzido por dois anos em indivíduos maiores de 75 anos, os autores concluíram também que os fatores mais associados com declínio na capacidade funcional foram o número de dias de atividades regulares, o número de refeições quentes diárias e o estado cognitivo, assim como, de acordo com outros estudos, o baixo nível de educação, saúde e depressão.

Outro conceito que nos parece relevante de estar sendo colocado, na perspectiva da capacidade funcional e a qualidade de vida do idoso, é a mobilidade, que sem dúvida é um elemento importante para manutenção da independência nesta etapa da vida. Segundo Patla e Shumway-Cook ${ }^{63}$, a mobilidade é a habilidade para se mover independentemente de um ponto para outro. Esta habilidade para se movimentar de forma segura e independente é parte fundamental das atividades básicas da vida diária (ABVD), como as atividades de higiene pessoal e de se vestir, assim como das atividades instrumentais da vida diária (AIVD), como ir às compras, ao banco, visitar amigos, ir ao cinema, lavar e cozinhar. Mas os autores tentam naquele artigo colocar uma nova visão da mobilidade, em que não somente seja reconhecida como a simples habilidade de an- dar, mas também as características de começar e parar, mudanças na direção, superfícies com diferentes propriedades geométricas e físicas e a execução simultânea de outras tarefas como falar, virar e olhar para outro objeto ou carregar objetos, na qual o indivíduo tem que ter a habilidade para se adaptar e modificar o andar em função de distúrbios, assim como os desafios esperados e inesperados da locomoção. Desta forma, a capacidade de movimentação seria considerada, não como o número de tarefas que o sujeito pode ou não realizar, mas sim pela amplitude de contextos ambientais nos quais estas tarefas possam ser desempenhadas.

A mobilidade é uma variável extremamente importante associada não somente com a qualidade de vida na terceira idade mas também com a longevidade. Neste sentido, Hirvensalo et al. ${ }^{64}$ analisaram a relação entre a mobilidade e o nível de atividade física com a mortalidade e a independência em 1.109 homens e mulheres de 65 a 84 anos de idade na avaliação inicial, realizada oito anos antes da segunda. A mobilidade foi definida como a habilidade para caminhar $2 \mathrm{~km}$ e subir um lance de escadas sem dificuldade. Os resultados mostraram claramente que as alterações na mobilidade e no nível de atividade física predizem a dependência e a morte em homens e mulheres maiores de 65 anos de idade. Os indivíduos com alterações da mobilidade tiveram um risco maior de morte e dependência do que aqueles que conseguiram manter a mobilidade. Os homens sedentários e com alteração da mobilidade tiveram sete vezes mais risco de perder a independência do que os ativos sem alteração; e nas mulheres esse risco foi quase quatro vezes maior. Com relação à mortalidade, esse risco foi três vezes maior em ambos os sexos. Mas o fato mais interessante mostrado ineditamente por este estudo foi que os sujeitos com alterações da mobilidade, mas que se mantiveram ativos, apresentaram menor risco de morte do que os sedentários, indicando que a atividade física foi um fator protetor de mortalidade em indivíduos com alterações da mobilidade.

De acordo com recente revisão realizada por Daley e Spinks ${ }^{65}$ sobre envelhecimento, mobilidade e exercício, os limiares de sensação cutânea e proprioceptiva se elevam com a idade, especialmente nos membros inferiores, reduzindo a percepção de vibração da articulação do joelho. Sendo o joelho a maior fonte de receptores que controlam a postura, esta perda pode diminuir consideravelmente o controle do equilíbrio e age como o principal responsável pela disfunção do andar nas pessoas idosas. De acordo com os autores, mulheres idosas que se exercitavam demonstraram menor desequilíbrio postural do que as sedentárias; quanto mais ativas, menor o grau de desequilíbrio. Da mesma forma, mulheres que participaram em atividades vigo- 
rosas por seis semanas a 10 anos mostraram ter um melhor equilíbrio do que mulheres sedentárias da mesma idade. $\mathrm{Na}$ mesma revisão $\mathrm{o}^{65}$ os autores analisaram o efeito do exercício na velocidade de andar com o envelhecimento. O exercício aeróbico e o treinamento de força têm mostrado um impacto positivo na amplitude de passada em homens idosos. No entanto, o exercício não influencia a velocidade de andar nos indivíduos idosos, exceto em um passo muito lento. Os autores apresentam evidências de que programas de exercício de cinco anos têm mostrado melhora na flexão e na rotação do quadril em mulheres de 50 a 71 anos. Provavelmente, além do exercício aeróbico e do treinamento de força, seja necessário um programa de treinamento específico da velocidade de andar para conseguir melhoras significantes nessa variável no indivíduo que está envelhecendo.

\section{DISCUSSÃO}

As evidências epidemiológicas apontam para um decréscimo do nível de atividade física com o aumento da idade cronológica, tornando o sedentarismo um fator de risco de morbidade e mortalidade durante o processo de envelhecimento. Os dados apontam que as barreiras para a prática de atividade física regular na terceira idade são facilmente superáveis e que estratégias de políticas públicas de saúde podem ser implantadas para superar a falta de equipamento, a falta de tempo e de conhecimento que são apontadas como as barreiras mais comuns. Dessa forma, é possível encorajar a adoção de um estilo de vida ativo durante o envelhecimento, sensibilizando a população sobre a possibilidade de ser fisicamente ativo sem precisar ter muito tempo e habilidades, conhecimentos ou equipamentos específicos. Do mesmo modo, as evidências enfatizam o papel do médico e da família como agentes facilitadores para a prática regular da atividade física nesta etapa da vida. Neste aspecto é fundamental considerar as observações realizadas recentemente por Christmas e Andersen ${ }^{66}$, que sugerem para os profissionais da área da saúde, especialmente os médicos, estratégias específicas para abordar e motivar o paciente idoso sedentário levando em consideração o padrão de atividade física do indivíduo durante a vida e seus interesses, assim como o nível de atividade física nos três últimos meses, o nível de interesse e motivação para o exercício e as preferências sociais para a prática da atividade física regular. Para reforçar e conseguir sucesso os autores recomendam que a prescrição da atividade física seja realizada por escrito, levando em conta o tempo disponível para realizar atividade física, o desejo do paciente, os equipamentos e as facilidades disponíveis, lembrando as comorbidades que podem afetar o desempenho para realizar atividade física e, o mais importante, ser esti- mulada como um hábito para o resto da vida. A avaliação médica, no entanto, deve ser utilizada com critérios específicos para converter esta ferramenta em mais uma barreira para a prática da atividade física. O último posicionamento a este respeito de um dos maiores especialistas da área, Shephard ${ }^{67}$, aponta claramente, com fortes evidências epidemiológicas, que o idoso que deseja realizar atividades físicas leves ou moderadas deve ser encorajado a se envolver regularmente sem uma avaliação médica especial, já que é desnecessária e um fator desmotivante. Devido às claras e fortes evidências epidemiológicas de que o estilo de vida ativo é um fator fundamental na prevenção, tratamento e controle das doenças crônicas não transmissíveis, a promoção da atividade física regular deve ser promovida nesta população com políticas claras e estratégias de implementação recentemente descritas por S. Matsudo e V. Matsudo ${ }^{68}$. As políticas públicas devem promover na população o estímulo à adoção da recomendação atual da atividade física, encorajando os indivíduos de todas as idades, mas em especial os maiores de 50 anos, a realizar pelo menos 30 minutos de atividade física moderada por dia, na maior parte dos dias, de forma contínua ou acumulada. Estas estratégias devem enfatizar a não obrigatoriedade da avaliação médica (com exceção dos casos sintomáticos) ou da participação em programas estruturados ou supervisionados para a adoção de um estilo de vida ativo como mecanismo de promoção da saúde.

\section{CONCLUSÕES}

As evidências epidemiológicas apresentadas nos permitem concluir que a atividade física regular e a adoção de um estilo de vida ativo são necessárias para a promoção da saúde e qualidade de vida durante o processo de envelhecimento. A atividade física deve ser estimulada não somente no idoso, mas também no adulto, como forma de prevenir e controlar as doenças crônicas não transmissíveis que aparecem mais freqüentemente durante a terceira idade e como forma de manter a independência funcional. As atividades que devem ser mais estimuladas são as atividades aeróbicas de baixo impacto, mas preferencialmente o exercício com pesos, para estimular a manutenção da força muscular dos membros superiores e inferiores, deve ser a prioridade no idoso. Da mesma forma, o equilíbrio e os movimentos corporais totais devem fazer parte dos programas de atividade física na terceira idade. As evidências sugerem que a atividade física regular e o estilo de vida ativo têm um papel fundamental na prevenção e controle das doenças crônicas não transmissíveis, especialmente aquelas que se constituem na principal causa de mortalidade: as doenças cardiovasculares e o câncer. Mas, além disto, a 
atividade física está associada também com uma melhor mobilidade, capacidade funcional e qualidade de vida durante o envelhecimento. É importante enfatizar, no entanto, que tão importante quanto estimular a prática regular

\section{REFERÊNCIAS}

1. Caspersen CJ, Kriska AM, Dearwater SR. Physical activity epidemiology as applied to elderly populations. Baillieres Clin Rheumatol 1994;8: 7-27.

2. Yusuf HR, Croft JB, Giles WH, Anda RF, Casper ML, Caspersen CJ, Jones DA. Leisure-time physical activity among older adults. Arch Intern Med 1996;156:1321-6.

3. Andrade EL, Matsudo SMM, Matsudo VKR, Araújo TL, Andrade DR, Oliveira LC, Figueira AJ. Barriers and motivational factors for physical activity adherence in elderly people in developing country [abstract]. Med Sci Sports Exerc 2000;33(Supl 7):141. [Presented at 47 $7^{\text {th }}$ American College of Sports Medicine Annual Meeting; 2000 Mai 31-Jun 3; Indianapolis (Indiana)].

4. Satariano WA, Haight TJ, Tager IB. Reasons given by older people for limitation or avoidance of leisure time physical activity. J Am Geriatr Soc 2000;48:505-12

5. Andrade EL, Matsudo SMM, Matsudo VKR, Araújo TL, Andrade DR, Figueira Jr. AJ, Oliveira LC. Nível de atividade física de adultos acima de 50 anos de idade do Estado de São Paulo. In: Anais XXII Simpósio Internacional de Ciências do Esporte; 1999 out 7-10. São Paulo, Brasil. Celafiscs, 1999:125.

6. Pate R, Pratt M, Blair SN, Haskell W, Macera CA, Bouchard C, et al Physical activity and public health: a recommendation from the Centers for Disease Control and Prevention and the American College of Sports Medicine. JAMA 1995;273:402-7.

7. Matsudo SM, Andrade EL, Matsudo VK, Araújo TL, Andrade DR, Figueira AJ, Oliveira LC. Nível de atividade física em relação ao grau de conhecimento do novo paradigma da atividade física em indivíduos maiores de 50 anos. In: Anais II Congresso Brasileiro de Atividade Física e Saúde; nov 24-26. Florianópolis, Brasil. NuPAF, 1999:85.

8. Oyama ER, Oliveira JG. Educação física e o idoso: implicações de gênero. Revista da APEF 1997;12:5-20.

9. Cousins SO, Keating N. Life cycle patterns of physical activity among sedentary and active older women. J Aging Phys Act 1995;3:340-59.

10. Young DR, Appel LJ, Jee SH, Miller ER. The effects of aerobic exercise and T'ai Chi on blood pressure in older people: results of a randomized trial. J Am Geriatr Soc 1999;47:277-84.

11. World Health Organization. WHO Guidelines for physical activity in older persons. Fourth International Congress of Physical Activity, Aging and Sports; 1996. August 27-31; Heidelberg, Germany; University of Heidelberg; 1996.

12. Secretaria de Estado da Saúde-Celafiscs. Manual do Programa Agita São Paulo. São Paulo (SP), 1998.

13. Ettinger WH, Mitchell BS, Blair SN. Should you see a doctor before you start exercising? Fitness after 50. $1^{\text {st }}$ ed. St. Louis: Beverly Cracom Publications, 1996:71-86.

14. Evans WJ. Exercise training guidelines for the elderly. Med Sci Sports Exerc 1999;31:12-7.

15. Pescatello LS, Dipietro L. Physical activity in older adults. Sports Med 1993;15:353-64.

16. Arent SM, Landers DM, Etnier JL. The effects of exercise on mood in older adults: a meta-analytic review. J Aging Phys Act 2000;8:407-30. da atividade física aeróbica ou de fortalecimento muscular, as mudanças para a adoção de um estilo de vida ativo no dia-a-dia do indivíduo são parte fundamental de um envelhecer com saúde e qualidade.

17. Kraemer AF, Hahn S, McAuley E. Influence of aerobic fitness on the neurocognitive function of older adults. J Aging Phys Act 2000;8:37985

18. Okuma SS. Investigando o significado da atividade física para o idoso. O idoso e a atividade física. $1^{\underline{a}}$ ed. Campinas: Papirus, 1998:111-9.

19. Faria Junior AG. Atividades físicas para idosos - um desafio para a educação gerontológica. In: Anais do $7^{0}$ Congresso de Educação Física e Ciências do Esporte dos Países de Língua Portuguesa; 1999 ago 26-30, Florianópolis, Brasil, 1999:119-28.

20. Feigenbaum MS, Pollock ML. Prescription of resistance training for health and disease. Med Sci Sports Exerc 1999;31:38-45.

21. Sparling PB, Cantwell JD. Strength training guidelines for cardiac patients. Physician Sports Med 1986;17:190-6.

22. McCartney N. Role of resistance training in heart disease. Med Sci Sports Exerc 1998;30(Suppl):S396-402.

23. American College of Sports Medicine. Position stand on exercise and physical activity for older adults. Med Sci Sports Exerc 1998;30:9921008.

24. Fiatarone MA. Physical activity and functional independence in aging. Res Q Exerc Sport 1996;67(Suppl 3):70.

25. Hurley BF, Hagberg JM. Optimizing health in older persons: aerobic or strength training? Exerc Sport Sci Rev 1998;26:61-90.

26. Yan JH, Downing JH. Tai Chi: an alternative exercise form for seniors. J Aging Phys Act 1998;6:350-62.

27. Yan JH. Tai Chi practice improves senior citizens' balance and arm movement control. J Aging Phys Act 1998;6:271-84.

28. Lan C, Lai JS, Chen S, Wong M. 12-month Tai Chi training in the elderly: its effect on health fitness. Med Sci Sports Exerc 1998;30:345-51.

29. Lan C, Chen S, Lai J, Wong M. The effect of Tai Chi on cardiorespiratory function in patients with coronary artery bypass surgery. Med Sci Sports Exerc 1999;31:634-8.

30. Oliveira RF, Matsudo SMM, Andrade DR, Matsudo VKR. Effect of TaiChi-Chuan on physical fitness of elderly women [abstract]. Med Sci Sports Exerc 1999;31(Suppl 5):385. [ Presented at $46^{\text {th }}$ American College of Sports Medicine Annual Meeting; 1999 Jun 2-5; Seattle (Washington)]

31. Oliveira RF, Matsudo SMM, Matsudo VKR. Efeito de um período de destreinamento após um programa de treinamento de Tai Chi Chuan sobre o nível de aptidão física de mulheres idosas. In: Anais XXII Simpósio Internacional de Ciências do Esporte; 1999 out 7-10. São Paulo, Brasil. Celafiscs, 1999:128.

32. Young DR, Appel LJ, Jee SH, Miller ER. The effects of aerobic exercise and T'ai Chi on blood pressure in older people: results of a randomized trial. J Am Geriatr Soc 1999;47:277-84.

33. Governo do Estado de São Paulo. Secretaria de Estado da Saúde. Programa Agita São Paulo. São Paulo (SP), 1998.

34. Murphy MH, Hardman A. Training effects of short and long bouts of brisk walking in sedentary women. Med Sci Sports Exerc 1998;30:1527.

35. Dunn AL, Marcus BH, Kampert JB, Garcia ME, Kohl HW, Blair SN. Comparison of lifestyle and structured interventions to increase physical activity and cardiorespiratory fitness. JAMA 1999;281:327-34. 
36. King AC, Rejeski WJ, Buchner DM. Physical activity interventions targeting older adults. A critical review and recommendations. Am J Prev Med 1998;15:316-33.

37. Chen W, Chang J, Pollock M, Graves JE, Probart CK, Splitter D. Effect of aerobic exercise training on anxiety reduction and health behaviors of healthy men and women 60 to 79 years of age. Res Q Exerc Sport 1992; 63(Suppl 1):33-8.

38. Stephens KE, Vanhuss WD, Olson HW, Montoye HJ. The longevity, morbidity and physical fitness of former athletes. Med Sci Sports Exerc 1990;22:742-50.

39. Paffenbarger RS. Contributions of epidemiology to exercise science and cardiovascular health. Med Sci Sports Exerc 1988;20:426-38.

40. Blair SN, Kohl HW, Paffenbarger Jr RS, Clark DG, Cooper KH, Gibbons LW. Physical fitness and all-cause mortality. JAMA 1989;262:2395401.

41. Lee IM, Hsieh CC, Paffenbarger Jr RS. Exercise intensity and longevity in men. JAMA 1995;273:1179-84.

42. Lissner L, Bengtsson C, Bjorkelund C, Wedel H. Physical activity levels and changes in relation to longevity. A prospective study of Swedish women. Am J Epidemiol 1996;143:54-62.

43. Kushi LH, Fee RM, Folsom AR, Mink PJ, Anderson KE, Sellers TA. Physical activity and mortality in postmenopausal women. JAMA 1997; 277:1287-92.

44. Paffenbarger Jr. RS, Lee IM. Physical activity and fitness for health and longevity. Res Q Exerc Sport 1996;67:11-28.

45. Farrell SW, Kampert JB, Kohl HW, Barlow CE, Macera CA, Paffenbarger Jr RS, et al. Influences of cardiorespiratory fitness levels and other predictors on cardiovascular disease mortality in men. Med Sci Sports Exerc 1998;30:899-905.

46. Paffenbarger Jr RS, Kampert JB, Lee IM. Physical activity and health of college men: longitudinal observations. Int J Sports Med 1997;18:S2003.

47. Wannamethee G, Shaper G, Walker M. Physical activity alterations, mortality and coronary disease prevalence in older men. Lancet 1998; 351:1603-8

48. Sihvonen S, Rantanen T, Heikkinen E. Physical activity and survival in elderly people: a five-year follow-up study. J Aging Phys Act 1998;6: $133-40$.

49. Hakim AA, Petrovitch H, Burchfiel CM, Ross WG, Rodriguez BL, White LR, et al. Effects of walking on mortality among nonsmoking retired men. N Engl J Med 1998;338:94-9.

50. Lee IM, Paffenbarger RS. Associations of light, moderate, and vigorous intensity physical activity with longevity. Am J Epidemiol 2000;151: 293-9.

51. Lee IM, Sesso HD, Paffenbarger RS. Physical activity and risk of lung cancer. Int J Epidemiol 1999;28:620-5.
52. Sesso HD, Paffenbarger RS, Ha T, Lee IM. Physical activity and cardiovascular disease risk in middle-aged and older. Am J Epidemiol 1999; 150:408-16.

53. Mensink GB, Ziese T, Kok FJ. Benefits of leisure-time physical activity on the cardiovascular risk profile at older age. Int J Epidemiol 1999; 28:659-66.

54. Evenson KR, Rosamond WD, Cai J, Toole JF, Hutchinson RG, Shahar E, Folsom AR. Physical activity and ischemic stroke risk. Stroke 1999;30: 1333-9.

55. Hu FB, Stampfer MJ, Colditz GA, Ascherio A, Rexrode KM, Willett WC, Manson JE. Physical activity and risk of stroke in women. JAMA 2000;283:2961-7.

56. Brill PA, Macera CA, Davis DR, Blair SN, Gordon N. Muscular strength and physical function. Med Sci Sports Exerc 2000;32:412-6.

57. Lee Y. The predictive value of self assessed general, physical, and mental health on functional decline and mortality in older adults. J Epidemiol Community Health 2000;54:123-9.

58. Laukkanen P, Leskinen E, Kauppinen M, Sakari-Rantala R, Heikkinen E. Health and functional capacity as predictors of community dwelling among elderly people. J Clin Epidemiol 2000;53:257-65.

59. Leveille SG, Guralnik JM, Ferrucci L, Langlois JA. Aging successfully until death in old age: opportunities for increasing active life expectancy. Am J Epidemiol 1999;149:654-64.

60. Vita AJ, Terry RB, Hubert HB, Fries JF. Aging, health risks, and cumulative disability. N Engl J Med 1998;338:1035-41.

61. Morey MC, Pieper CF, Cornoni-Huntley J. Is there a threshold between peak oxygen uptake and self-reported physical functioning in older adults? Med Sci Sports Exerc 1998;8:1223-9.

62. Hébert R, Brayne C, Spiegelhalter D. Factors associated with functional decline and improvement in a very elderly community-dwelling population. Am J Epidemiol 1999;150:501-10.

63. Patla AE, Shumway-Cook A. Dimensions of mobility: defining the complexity and difficulty associated with community mobility. J Aging Phys Act 1999;7:7-19.

64. Hirvensalo M, Rantanen T, Heikkinen E. Mobility difficulties and physical activity as predictors of mortality and loss of independence in the community-living older population. J Am Geriatr Soc 2000;48:493-8.

65. Daley MJ, Spinks WL. Exercise, mobility and aging. Sports Med 2000;29:1-12.

66. Christmas C, Andersen RA. Exercise and older patients: guidelines for the clinician. J Am Geriatr Soc 2000;48:318-24.

67. Shephard RJ. Does insistence on medical clearance inhibit adoption of physical activity in the elderly? J Aging Phys Act 2000;8:301-11.

68. Matsudo SM, Matsudo VK. Physical activity and ageing in developing countries. Perspectives 2000;2:65-84. 